\title{
Dessine-moi un mouton : contraintes descriptives et liberté rhétorique dans les publicités à caractère scientifique ou technique
}

\section{Marie-Hélène Fries-Verdeil}

\section{(2) OpenEdition}

\section{Journals}

Édition électronique

URL : http://journals.openedition.org/asp/4322

DOI : $10.4000 /$ asp. 4322

ISSN : 2108-6354

Éditeur

Groupe d'étude et de recherche en anglais de spécialité

\section{Édition imprimée}

Date de publication : 1 octobre 1993

Pagination : 355-369

ISSN : 1246-8185

\section{Référence électronique}

Marie-Hélène Fries-Verdeil, « Dessine-moi un mouton : contraintes descriptives et liberté rhétorique dans les publicités à caractère scientifique ou technique », ASp [En ligne], 2 | 1993, mis en ligne le 06 mars 2014, consulté le 19 avril 2019. URL : http://journals.openedition.org/asp/4322; DOI : 10.4000/ asp.4322

Ce document a été généré automatiquement le 19 avril 2019.

Tous droits réservés 


\title{
Dessine-moi un mouton : contraintes descriptives et liberté rhétorique dans les publicités à caractère scientifique ou technique
}

\author{
Marie-Hélène Fries-Verdeil
}

1 Cette communication sur les contraintes et les libertés de la publicité à caractère scientifique ou technique ${ }^{1}$ est née de la nécessité pratique de trouver des documents permettant de faire parler les étudiants scientifiques sur des sujets proches des matières qu'ils étudient, tout en se prêtant à une exploitation plus large, par exemple éthique ou culturelle, qui puisse les amener à développer leur sens critique et leur réflexion personnelle. La publicité offre un terrain de choix dans cette perspective, mais exige, pour une utilisation pédagogique fructueuse, quelques outils d'analyse. La rhétorique, qui connaît une renaissance certaine depuis les années soixante, constitue l'un de ces outils. Cette communication essaye donc de brosser, dans un premier temps, un tableau rapide des contraintes descriptives qui pèsent sur la publicité à caractère scientifique et technique, ce qui permet de définir une norme, celle d'une publicité en noir et blanc, à fonction référentielle et pédagogique, qui montre pour démontrer. On verra ensuite comment cette norme est battue en brèche par la rhétorique, pour tenter d'analyser enfin, à partir de l'étude de la synecdoque, de la métonymie et de la métaphore, comment la rhétorique ouvre la porte à une multiplication du sens, et à une libération de l'imaginaire.

\section{Contraintes descriptives}

Dans la grande majorité des publicités à caractère scientifique et technique, l'image se limite à montrer l'objet dont on veut vanter les mérites, présenté de face, en noir et blanc sur fond vide, sans arrière-plan, sans personnages accompagnateurs (cf. l'exemple d'un goniomètre pour déterminer les structures cristallines par rayons $\mathrm{X}$, en annexe 1). 
L'image a alors une fonction analogique et une valeur référentielle. On assiste à une autoprésentation de l'objet. On atteint alors une sorte de degré zéro de l'image, qui se distingue également des couleurs voyantes de la publicité pour produits de grande consommation, et des effets esthétiques de la publicité pour produits de luxe. Là où l'image se borne à être simple illustration, le texte publicitaire se veut souvent pure démonstration. Le message, souvent substantiel, est centré sur un produit, ou une gamme de produits, dont il énumère longuement les caractéristiques. Et l'acheteur potentiel se voit expliquer tous les avantages d'utilisation qui pourraient en résulter pour lui : facilité, rapidité, puissance, moindre coût éventuellement. Le message référentiel se double donc d'un message d'implication du destinataire, dont la fonction psychologique principale est de promouvoir la réussite professionnelle par l'économie d'efforts, de temps ou d'argent.

3 La publicité scientifique se définit donc comme un genre caractérisé par le noir et blanc, utilisé de préférence à la couleur, par une image à vocation illustrative, un texte copieux à fonction informative, une implication minimale des sentiments du destinataire, et enfin un message qui s'intéresse à l'être plutôt qu'à la valeur, qui se veut ontologique avant d'être axiologique.

Cette norme informative austère est probablement due à la double spécificité des produits considérés et du public visé. Les créations de la technique sont souvent des machines ou des appareillages complexes, si bien que vanter leurs mérites revient souvent à rédiger un mini-mode d'emploi. Un public de techniciens et de scientifiques, d'autre part, n'est que rarement libre d'effectuer des achats d'impulsion : le plus souvent, il faut en référer à un supérieur hiérarchique, qui se laissera d'autant plus aisément convaincre que les arguments seront rationnels et les détails précis.

\section{Liberté rhétorique}

5 Cette analyse ne tient cependant pas compte du jeu de la concurrence. Pour vendre, même dans le monde de la science et de la technique, il faut non seulement informer, mais encore se distinguer du lot. La raison d'être de toute publicité, c'est de se faire remarquer. "Esse est percipi », comme le note très justement Georges Péninou dans son Intelligence de la publicité (1973: 179)². Le but de tout publicitaire est d'arriver par là à éveiller chez les clients potentiels le désir d'en savoir plus, pour les transformer en acheteurs effectifs. Dans ce contexte, on comprend que, sous la norme d'une publicité informative en noir et blanc, fournissant un point de vue objectif à des gens sérieux, se cachent souvent les ressources variées de la persuasion.

6 Voici par exemple une publicité pour la firme Oxford Instruments, qui travaille dans les domaines du magnétisme et des basses températures, et fabrique des appareils permettant de faire des expériences sur les semi-conducteurs (cf. annexe 2). L'image, en noir et blanc, représente un circuit imprimé de taille légèrement supérieure à la grandeur nature en arrière-plan, et des puces électroniques, prêtes à être enfichées dans ce circuit imprimé, dans le plan médian. Le couvercle de plastique qui recouvre d'habitude ces puces électroniques a été ôté, de manière à faire apparaitre les semi-conducteurs et les connexions électriques. Le premier plan est occupé par la représentation filaire d'un buste humain présenté de trois quarts, tourné vers le lecteur. Le choix privilégié d'une tête désigne le cerveau par métonymie, le contenant représentant le contenu. Il désigne donc le siège de la pensée et semble la répétition sur le plan de l'image de la répétition 
anaphorique impérative du slogan : think, think, think. La représentation filaire, quant à elle, établit une analogie de forme entre la résille de lignes formant la tête pensante et le circuit électronique en arrière-plan. Une analogie de substance se trouve suggérée par là, comme si le buste concentrait son activité pensante sur l'imagination et la conception de nouveaux produits électroniques. Parallèlement, l'effet du grossissement hyperbolique du circuit imprimé en arrière-plan, par rapport au buste en premier plan, vient renforcer cette idée, comme si la préoccupation de nouvelles inventions électroniques restait continuellement à l'arrière-plan du cerveau pensant, qui représente donc, on l'a deviné maintenant, un client potentiel de la firme Oxford Instruments, un chercheur travaillant sur les semi-conducteurs. D'ailleurs, le raccourcissement du nom de la firme en Oxford semble une garantie supplémentaire de son sérieux, comme si elle s'identifiait par double sens au centre de savoir et d'excellence qu'est l'université du même nom.

7 Métonymie, anaphore, hyperbole, double sens, quatre figures de rhétorique au moins ont été mobilisées pour faire passer le message : « achetez chez Oxford Instruments », et l'art de la persuasion semble ici aller de pair avec le souci de l'information. La rhétorique, par ses figures verbales ou visuelles, vient donc rompre la norme de la publicité scientifique et technique, fondée sur une stricte dénotation. Dans le cadre limité de cette communication, il ne sera pas possible d'étudier toutes les libertés prises grâce à la rhétorique ${ }^{3}$. Nous nous limiterons maintenant à la rhétorique de l'image et, plus précisément, aux tropes, c'est-à-dire aux figures de rhétorique jouant sur un transfert de sens : métaphores, métonymies et synecdoques. En effet, ce sont elles qui permettent la rupture la plus décisive avec la norme, et sont donc le plus clairement source de liberté.

\section{Synecdoque : I'explosion du sens}

8 Si l'on se souvient qu'en rhétorique classique la figure de la synecdoque se définit d'habitude par la formule "la partie pour le tout», on imagine sans peine que l'image publicitaire puisse faire deviner, à travers un détail significatif, tout un ensemble. Le corps humain représente un domaine d'application de choix pour les synecdoques. On a déjà vu comment un buste en représentation filaire pouvait représenter un client efficace et passionné de la firme Oxford Instruments. Le fabricant britannique d'ordinateurs Gould a plutôt choisi une main habillée d'un gant de boxe, et levée en signe de victoire, pour signifier sa position de force dans le marché des ordinateurs travaillant sous système d'exploitation Unix (cf. annexe $\mathrm{n} 3$ ). Le soin d'imaginer un ring de boxe, son suspense et ses coups, est laissé au lecteur, et c'est ce qui fait la force de l'image. D'autre part, la pancarte brûlant à l'arrière-plan, qui porte en grosses lettres le mot « Unix », et représente, à nouveau par synecdoque, tous les ordinateurs départementaux travaillant sous ce système d'exploitation, ainsi que le feu rongeant la pancarte, rappellent au lecteur la prééminence de la firme Gould sur tous ses concurrents. D'ailleurs, même si les flammes de l'arrière-plan n'y suffisaient pas, la main qui tient au premier plan le poignet du boxeur, et laisse deviner la manche d'une chemise blanche et d'un costume sombre de coupe impeccable, n'aurait pas manqué d'attirer l'attention du lecteur, par une troisième synecdoque, sur tous les clients respectables et au succès économique certain, déjà satisfaits par les ordinateurs Gould. 


\section{Métonymie : la surprise du sens}

9 De toutes les figures de rhétorique, la métonymie est celle qui a la définition la plus floue. On considère généralement qu'elle est liée à une relation de proximité, par exemple, l'effet remplaçant la cause ou le lieu de fabrication le produit. Le glissement métonymique est souvent lié, en publicité, à un effet de mystère. Prenons par exemple la campagne lancée par IBM en 1986 pour persuader le public de la convivialité de leurs micro-ordinateurs. L'une de leurs publicités représente une tomate toute fraîche, sur laquelle perlent encore deux gouttes d'eau ou de rosée, énigmatiquement posée sur du marbre blanc veiné de bleu (cf. annexe 4). Le dessin coloré de la tomate a pour pendant, dans l'accroche, les signes phonétiques donnant les prononciations britanniques et américaines du mot "tomate " en anglais. Mais le mystère reste pour l'instant aussi entier : quel est le rapport entre une tomate et la firme IBM ? La solution est donnée par le texte de la publicité, qui explique qu'IBM est en train de participer à la nouvelle édition du prestigieux Oxford English Dictionary (OED), mettant gratuitement ordinateurs et techniciens à la disposition des compilateurs. On comprend alors que la prononciation du mot "tomate", ainsi que sa traduction visuelle, signifient, par synecdoque, tout l'ensemble de l'OED, et par le jeu métonymique qui substitue le contenant au contenu, l'OED maintenant informatisé par les techniciens d'IBM représente à son tour les ordinateurs qui le contiennent. La tomate signifie donc bien IBM, par synecdoque et métonymie interposées. D'autant qu'elle peut aussi être lue comme une allusion perfide à la firme rivale Apple, le numéro un de l'informatique étant à son concurrent Macintosh ce que la pomme d'or est à la pomme vulgaire... La métonymie crée donc un effet de surprise, et appelle le lecteur à s'arrêter, pour partir en quête de sens et satisfaire sa curiosité.

\section{Métaphore : quand le sens devient symbole}

10 De toutes les figures de style, la métaphore, ou comparaison privée de son terme comparatif, est certainement la plus connue et la plus féconde. Elle a toujours gardé une place de choix en littérature, même au plus fort de la disgrâce de la rhétorique antique. La raison en est simple : par le jeu de la similitude, une métaphore permet de représenter concrètement un terme abstrait, de passer du concept à l'analogie, du règne de la démonstration à celui de l'imagination.

11 La publicité scientifique joue souvent sur les métaphores d'usage, en utilisant leur représentation visuelle pour renforcer l'effet escompté. Voici l'exemple de deux publicités où les slogans sont construits sur la métaphore de la camisole de force, pour signifier contraintes et limitations. La première émane d'un fabricant de polyuréthannes, la seconde d'un concepteur de logiciels (cf. annexes 5 et 6). L'effet produit est plus grand dans le second cas, parce que la métaphore verbale se double d'une illustration visuelle. Les contraintes du système d'exploitation DOS, comparées à la facilité d'utilisation de la souris, sont alors mises en valeur par l'air pitoyable de l'utilisateur en col blanc, empêché d'utiliser son logiciel « Windows » par une camisole de force sur laquelle sont inscrits les noms de différents programmes sous DOS.

$12 \mathrm{Si}$, dans les métaphores d'usage, le rôle de l'image se borne souvent à être simple illustration du jeu verbal, pour le rendre plus concrètement encore, dans les métaphores 
d'invention par contre son rôle est prépondérant, d'autant plus que le jeu de similarités et de différences sur lequel sont bâties les métaphores permet une infinité d'illustrations possibles. En effet, pour inventer une métaphore en publicité, il suffit de décider ce qu'il faut mettre en valeur parmi les caractéristiques du produit à promouvoir, et de trouver un autre objet qui possède cette même caractéristique. Soit par exemple une publicité pour les photocopieurs Minolta ayant choisi comme slogan : "There are times when faster is decidedly better" (cf. annexe 7). L'accent est résolument mis ici sur la rapidité, et l'utilité de ce gain de temps pour le client. L'image fournit une mise en scène dramatique du prix inestimable de la vitesse, avec le monstre du Loch Ness sortant des profondeurs derrière deux embarcations, un canot à rames et un puissant hors-bord. Le hors-bord, on l'a deviné, symbolise le photocopieur Minolta, dont il partage la rapidité, ce qui lui permet de doubler de vitesse un monstre dont le gigantisme fait écho aux « monstrous copying jobs " auquel le client se trouve parfois confronté. Quant au canot à rames, il renvoie sûrement aux photocopieurs concurrents ou plus anciens, moins performants en tout cas. L'élément métaphorisant (le hors-bord) est donc subordonné en principe à l'élément métaphorisé (le photocopieur), et placé au service du message publicitaire, dont il illustre le point principal (ici, la rapidité). Il s'en émancipe pourtant dans la mesure où l'élément métaphorisé est exclu de l'image principale, libérant l'imagination du lecteur pour rêver au monstre du Loch Ness, figure mythique émergée de l'eau primordiale de l'inconscient collectif.

On voit bien, à partir de cet exemple, pourquoi les métaphores visuelles apparaissent, pour la publicité scientifique et technique, comme une porte ouverte sur la liberté de lecture. Elles brisent le carcan de la représentation analogique en reléguant l'objet métaphorisé au second plan, permettant au sens de passer à travers les symboles, laissant libre cours au rêve et à l'imaginaire, tout en étant totalement extérieures au texte publicitaire, qui ne perd donc rien de son caractère descriptif et technique.

\section{Conclusion}

La publicité à caractère scientifique et technique connaît donc des contraintes bien délimitées, mais antagonistes : la nécessité d'informer le plus complètement possible ne va pas toujours de pair avec la persuasion qui est de mise pour présenter un produit comme le meilleur, le plus performant, etc. Les figures de rhétorique, grâce à leur double niveau simple et figuré, permettent de sortir du dilemme en créant du sens à partir des objets exposés : un modeste circuit imprimé, grâce à un agrandissement hyperbolique, se met alors à signifier la passion de la découverte dans ce domaine, une tomate campagnarde se gorge de tout le trésor de connaissances contenu dans l'oxford English Dictionary, et la rapidité d'un photocopieur évoque le surgissement du monstre du Loch Ness à battre de vitesse. Grâce à la rhétorique, la publicité de l'objet devient publicité de la valeur, l'information cède le pas à l'évocation, et la pédagogie s'efface pour ouvrir la porte à l'imaginaire. Et, de même que le Petit Prince de Saint-Exupéry, demandant à l'aviateur : "Dessine-moi un mouton », ne se satisfait vraiment que de la représentation d'une caisse percée, qui lui permet d'imaginer, à travers les trous, le mouton de ses rêves, de même un produit publicitaire n'est jamais plus désirable que lorsque les figures de rhétorique permettent à l'imaginaire de relayer la représentation, et par là même de la reléguer au second plan. 


\section{BIBLIOGRAPHIE}

Barthes, Roland. 1964. « Rhétorique de l'image ». Communications 4, 40-51.

Durand, Jacques. 1970. « Rhétorique et image publicitaire ». Communications 15, 71-93

Greven, Hubert. 1982. La langue des slogans publicitaires en anglais contemporain. Paris : Presses

Universitaires de France.

Péninou, Georges. 1972. Intelligence de la publicité : étude sémiotique. Paris : Robert Laffont.

\section{ANNEXES}

\section{Annexe 0. Compte-rendu des discussions}

Les trois thèmes principaux ont été le jeu de l'intertextualité publicitaire, l'ambiguïté de la métaphore, et la richesse de son détournement pédagogique. La publicité pour IBM (une tomate posée sur du marbre) a suscité plusieurs réactions. Jean-Louis Trouillon a rappelé que sa date de création (1986) renvoyait en écho au duel IBM-Apple de 1984, et Michel Perrin que le choix de la tomate, ou pomme d'or, en faisait un clin d'œil au logo de la marque rivale Macintosh, ou encore, selon la formule de Kathryn English « une pierre dans le jardin d'Apple ». Cette publicité est aussi signe de l'emprise mondiale d'IBM, qui incorpore à la fois l'Angleterre et les États-Unis à travers la tomate, et ses prononciations habituelles des deux côtés de l'Atlantique. Hubert Greven a vu un bel exemple d'intertextualité publicitaire, puisque les publicités se répondent l'une l'autre, non seulement à l'intérieur d'une même marque, mais aussi d'une marque à l'autre dans le cadre de la publicité comparative, aux États-Unis par exemple.

Hubert Greven nous a fait réfléchir sur l'ambiguïté de la rhétorique publicitaire en montrant que, si la métaphore visuelle ouvre le champ des possibilités de l'imaginaire, elle n'en renvoie pas moins au texte, qui lui au contraire clôt et emprisonne le lecteur dans le rôle d'acheteur potentiel, souvent à l'aide de différents procédés phoniques. Michel Perrin s'est interrogé sur la nature du processus métaphorique. La magie du Petit Prince, c'est, à partir d'une histoire toute simple, d'ouvrir sur quelque chose de beaucoup plus vaste. La caisse, c'est l'illustration de la liberté gagnée à travers l'acceptation de la contrainte, et le passage par une interprétation.

Thérèse Brouat a conclu en ouvrant une perspective de détournement pédagogique, et a expliqué comment créer, à partir de l'impact produit par une publicité, un deuxième impact linguistique, en utilisant l'énigmatique, dans l'accroche en particulier.

\section{Annexes 1 à 7 (voir fichier)}




\section{NOTES}

1. 1 C'est-à-dire toute publicité ayant pour objet, soit une machine ou un appareil, soit un aspect particulier des sciences et techniques.

2. Élaboration d'une métaphysique de l'image publicitaire à partir des catégories de la grammaire traditionnelle. Voir en particulier 165-173.

3. 3 Le lecteur désireux d'approfondir davantage pourra se référer en particulier à l'article de Jacques Durand, (1970), où il trouvera un tableau assez complet des figures de style et de leurs transpositions visuelles. Pour ce qui est du texte, la thèse d'Hubert Greven (1982) fournit une étude détaillée des figures de style dans les accroches. Voir en particulier le chapitre 4 : Niveau phonique 185-240, et le paragraphe sur l'activité métaphorique, 304-309.

\section{RÉSUMÉS}

La publicité à caractère scientifique ou technique cherche à vendre en informant et la description du produit (exposé des caractéristiques, démonstration visuelle) y est prépondérante. On serait tenté de définir ici un "degré zéro de la publicité", plus proche de la technicité d'un mode d'emploi que des subtilités d'une campagne d'affichage. Est-ce à dire pourtant que l'imaginaire n'y ait aucune place ? Non. Il faut certes informer pour vendre les produits techniques, mais cela ne suffit pas toujours à les démarquer de la concurrence. Pour passer de la promotion d'un objet à la création et à la libération du sens, synecdoques, métaphores et métonymies apparaissent comme des outils de choix.

Technical or scientific advertising aims at informing in order to sell, so that it focuses on the description of the product, with illustrative photographs and explanations of characteristics. This stern didactic norm is, however, often countered by the laws of competition. In order to sell well, a product has to be perceived as different. This is often successfully achieved through visual imagery and rhetoric. Thanks to synecdoche, metonymy and metaphor, explanations give way to imagination and the creation of meaning.

\section{INDEX}

Keywords : advertising, metaphor, metonymy, synecdoche

Mots-clés : publicité, métaphore, métonymie, synecdoque

\section{AUTEUR}

\section{MARIE-HÉLÈNE FRIES-VERDEIL}

Université Joseph Fourier, Grenoble 1. Marie-Helene.Fries@ujf-grenoble.fr 